\title{
Fluorescence in situ hybridization analysis is a helpful test for the diagnosis of dermatofibrosarcoma protuberans
}

\author{
Marie Karanian ${ }^{1,2,3}$, Gaëlle Pérot ${ }^{1,2}$, Jean-Michel Coindre ${ }^{1,2,3}$, Frédéric Chibon ${ }^{1,2}$, \\ Florence Pedeutour ${ }^{4}$ and Agnès Neuville ${ }^{1,2,3}$ \\ ${ }^{1}$ Department of Pathology, Institut Bergonié, Bordeaux, France; ${ }^{2}$ INSERM U916, Bordeaux, France; \\ ${ }^{3}$ Université de Bordeaux, Bordeaux, France and ${ }^{4}$ Laboratoire de Génétique des Tumeurs Solides, Institute for \\ Research on Cancer and Aging (IRCAN), University Hospital of Nice, Bordeaux, France
}

\begin{abstract}
Cytogenetically, most dermatofibrosarcoma protuberans are characterized by chromosomal rearrangements resulting in the collagen type-1 alpha 1 (COL1A1)-platelet-derived growth factor $\beta$ (PDGFB) fusion gene. This abnormality can be detected by fluorescence in situ hybridization (FISH) analysis in routine practice. The aim of this study was to evaluate the role of the FISH analysis in the diagnosis of dermatofibrosarcoma protuberans. A FISH analysis was prospectively and systematically performed on a series of 448 consecutive tumor specimens. All cases were reviewed by two independent pathologists and classified in three categories according to the probability of a DFSP diagnosis before molecular analyses. Cases were classified as certain when dermatofibrosarcoma protuberans was the only possible diagnosis. Those cases for which dermatofibrosarcoma protuberans remained the first diagnosis, but other differential diagnosis existed, were regarded as probable. When dermatofibrosarcoma protuberans was considered a differential diagnosis, they were labeled as possible. The final diagnosis was supported by clinicopathological findings and results of FISH analyses. Immunohistochemical analysis of CD34 was systematically performed, and additional markers when necessary. The cases $(n=37)$ with a non-interpretable FISH were excluded. For the 185 certain tumors specimens: 178 (96\%) FISH analyses showed a PDGFB/COL1A1 rearrangement, 7 (4\%) were negative. For the 114 probable tumors specimens: $104(91 \%)$ FISH analyses were positive and $10(9 \%)$ were negative leading to a new diagnosis in 8 cases. For the 112 possible cases: $91(81 \%)$ FISH analyses were negative and 21 (19\%) were positive. Of the 21 cases, initial diagnoses included unclassified sarcoma, myxofibrosarcoma, dermatofibroma, reactive lesion, solitary fibrous tumor, perineurioma, benign nerve sheath tumor, and undifferentiated spindle cell tumor without malignant evidence. FISH analysis has been helpful for confirming the diagnosis of dermatofibrosarcoma protuberans in $25 \%(104 / 411)$ of cases and necessary for the diagnosis of dermatofibrosarcoma protuberans in $5 \%(21 / 411)$ of cases.
\end{abstract}

Modern Pathology (2015) 28, 230-237; doi:10.1038/modpathol.2014.97; published online 1 August 2014

Dermatofibrosarcoma protuberans is a rare cutaneous/ subcutaneous mesenchymal tumor of intermediate malignancy. ${ }^{1}$ Dermatofibrosarcoma protuberans usually affects young to middle-aged adult, but it has been observed also in children and in the elderly. This lesion can present as nodular, multinodular, or

Correspondence: Professor J-M Coindre, MD, Department of Pathology, Institut Bergonié, 229 cours de l'Argonne, F-33000 Bordeaux, France.

E-mail: j.coindre@bordeaux.unicancer.fr

This study has been presented in abstract form at the 103rd Annual Meeting of the United States and Canadian Academy of Pathology, San Diego, March 2014.

Received 8 April 2014; revised 17 May 2014; accepted 18 May 2014; published online 1 August 2014 plaque mass, and it typically grows slowly. Although rarely metastasizing, dermatofibrosarcoma protuberans is locally aggressive and characteristically has a high recurrence rate that requires a surgical resection with wide tumor-free margins. For patients with advanced tumors or for cases that are difficult to remove surgically, a targeted therapy (inhibitor of tyrosine kinase) has proven to be beneficial. ${ }^{1-3}$

The diagnosis of dermatofibrosarcoma protuberans is mainly supported by clinical context, morphological appearance, and immunohistochemical profile. Histologically, this tumor is characterized by an infiltration in the dermis and subcutis along fibrous septa between adnexa and fat lobules, often 
resulting in a honeycomb appearance. A storiform or whorled pattern and uniform spindle cell tumor with elongated wavy nuclei and fewer than five mitoses in 10 high-power fields have often been described. Tumor cells in dermatofibrosarcoma protubrans are generally positive for CD34 staining. When the histological features are typical and the positivity of the CD34 staining is clear, the diagnosis is easy.

Nonetheless, unusual aspects such as myxoid, pigmented, plaque-like, epithelioid, giant cell fibroblastoma, as well as the fibrosarcomatous transformation that this tumor can undergo, sometimes without areas of classical dermatofibrosarcoma protuberans, ${ }^{1,4}$ render the diagnosis of dermatofibrosarcoma protuberans problematic. Moreover, it is increasingly commonplace to conduct a diagnosis on small specimens with insufficient histological criteria.

Recently, a chromosomal rearrangement was identified cytogenetically ${ }^{5-7}$ and described in $74-96 \%$ of dermatofibrosarcoma protuberans. ${ }^{8-12}$ This abnormality involves the chromosomes 17 and 22, and forms the fusion gene collagen type-1 alpha 1 (COL1A1)platelet-derived growth factor $\beta$ (PDGFB). ${ }^{1,5,13-14}$ This rearrangement can be detected by fluorescence in situ hybridization (FISH) analysis. ${ }^{8}$

It is now well established that a high proportion of sarcomas is associated with specific cytogenetic lesions. In such instances, molecular testing can be extremely useful to support challenging diagnoses of sarcoma. ${ }^{15-17}$

The aim of this study was to evaluate the potential contribution of FISH analysis in the diagnosis of dermatofibrosarcoma protuberans in a series of 448 cases, including specimens that were straightforward and others that were more difficult to determine. In addition, we tested the specificity of the CD34 expression for dermatofibrosarcoma protuberans and listed the differential diagnoses of dermatofibrosarcoma protuberans.

\section{Materials and methods}

\section{Selection of Cases}

The series consisted of 448 consecutive tumor specimens that were suspected to be a dermatofibrosarcoma protuberans according to accepted conventional diagnostic techniques (that is, histology and immunohistochemistry). These specimens were sent either to one of the authors (J-MC) in consultation or for a systematical central review and recording into the RRePS database (Réseau de Référence en Pathologie des Sarcomes https://rreps. sarcomabcb.org) from October 2007 to February 2013. A systematic FISH analysis was prospectively performed on the corresponding fixed embedded tissue specimens. Ninety-five of these cases have already been reported in a previous publication. ${ }^{17}$

Patient characteristics and clinical information on tumor location were retrieved from hospital records and referring pathologists. Thirty-two (8\%) specimens were obtained via punch biopsy, 42 (9\%) via shaving biopsy and $374(83 \%)$ via resection.

Four hundred and eleven samples (92\%) were fixed in buffered formalin and the remaining $37(8 \%)$ in other fixation fluids including AFA and Bouin of Holland. All paraffin-embedded sections were stained with hematoxylin and eosin.

\section{Histological Review and Classification}

All cases were reviewed by two pathologists and classified according to the probability of a dermatofibrosarcoma protuberans diagnosis as certain, probable, and possible. A tumor specimen was classified as a certain dermatofibrosarcoma protuberans when the following five criteria were met: (I) infiltration of the dermis and subcutis, (II) uniform spindle cells with minimal cytological atypia, (III) an architecture displaying a monotonous storiform pattern, (IV) proliferation along the connective tissue septa and between adnexae, interdigitation with fat lobules (honeycomb effect), (V) and a positive CD34 staining. ${ }^{1,4}$ A tumor specimen was classified as a probable dermatofibrosarcoma protuberans when the first contemplated diagnosis was dermatofibrosarcoma protuberans, but a differential diagnosis arose with some other tumor, such as cellular dermatofibroma. A tumor specimen was classified as possible dermatofibosarcoma protuberans when the diagnosis of dermatofibrosarcoma protuberans was not the first contemplated diagnosis or when the diagnosis of dermatofibrosarcoma protuberans was strongly confronted by another histological subtype.

The final diagnosis was supported by clinicopathological findings and the result of FISH analysis.

\section{Immunohistochemistry Analyses}

For immunohistochemical analysis, tissue sections were deparaffinized in xylene, hydrated in alcohol, and baked in microwave for $20 \mathrm{~min}$. Endogenous peroxydase was blocked and staining was performed on the Benchmark ultra-automated stainer (Ventana), using diaminobenzidine as chromogen. Anti-CD34 antibody (QBEnd/10; Ventana) staining was performed systematically and when necessary, additional markers were tested.

\section{Molecular Analyses}

FISH assay was performed using the Histology FISH accessory kit (Dako) according to the manufacturers' instructions.

COL1A1/PDGFB fusion and PDGFB rearrangement were detected using home-made probes. Specifically, for the identification of the COL1A1/PDGFB fusion, two BAC clones covering COL1A1 (RP11-93L18, RP-11893F2; red signals) and two BAC clones covering PDGFB (RP11642F17, RP11-959K5; green signals) were used, whereas $P D G F B$ rearrangement was detected using the hybrid of 
three BAC clones covering PDGFB (RP11-101B10, RP11434E5, RP11-1149B8). ${ }^{18}$

Green and red fluorescent signals were analyzed in regions of cellular tumor using a Nikon Eclipse 80i fluorescence microscope with appropriate filters. Pictures were captured using a Hamamatsu C4742-95 CCD camera and analyzed with the Genikon software (Alphelys, France). With the dual-color fusion probe, the COL1A1/ $P D G F B$ fusion was present when a yellow or a juxtaposed red-green signal was observed in the nucleus. A $P D G F B$ rearrangement was detected when red signals and green signals were separated in the nucleus with dual color break apart. A COL1A1-PDGFB fusion gene or a $P D G F B$ rearrangement was considered as present if at least $10 \%$ of cells' tumor showed a fusion or a rearrangement pattern as previously described.

\section{Results}

A total of 448 cases were included in this study. Two hundred cases (45\%) were classified as certain, 122 $(27 \%)$ as probable, and $126(28 \%)$ as possible before performing the molecular testing.

The median age of the patients was 45 years (range 1-89 years), with a female to male ratio of 1:1. The sites involved were: the trunk wall in 168 cases (37\%), the lower and upper limb in 112 (25\%) and in 98 cases $(22 \%)$, respectively, the head and neck in 42 cases $(10 \%)$, and the genital area in 5 cases (1\%). Twenty-three (5\%) tumors occurred in unknown sites.

Overall, 303 (68\%) FISH analyses revealed a COL1A1/PDGFB rearrangement, whereas 108 $(24 \%)$ cases tested negative (Table 1$)$.

\section{Non-interpretable FISH Cases}

The results of 37 (8\%) FISH analyses were noninterpretable, the main reason being the use of a fixative other than formalin. In this situation, the first diagnosis was retained. These tissue samples, from 19 females and 18 males, were obtained by resection in $34(92 \%)$ cases, shave biopsy in $2(6 \%)$ cases, and punch biopsy in $1(2 \%)$ case. Thirty-five

Table 1 Proportion of FISH positivity according to degree of certainty in the diagnosis of dermatofibrosarcoma protuberans (percentage of FISH-interpretable cases only)

\begin{tabular}{lrrrr}
\hline $\begin{array}{l}\text { Degree of } \\
\text { certainty of } \\
\text { DFSP }\end{array}$ & $\begin{array}{c}\text { FISH } \\
\text { positive } \\
(\%)\end{array}$ & $\begin{array}{c}\text { FISH } \\
\text { negative } \\
(\%)\end{array}$ & $\begin{array}{c}\text { Non- } \\
\text { interpretable }\end{array}$ & Total \\
\hline Certain DFSP & $178(96)$ & $7(4)$ & 15 & 200 \\
Probable DFSP & $104(91)$ & $10(9)$ & 8 & 122 \\
Possible DFSP & $21(19)$ & $91(81)$ & 14 & 126 \\
Total & $303(74)$ & $108(26)$ & 37 & 448 \\
\hline
\end{tabular}

Abbreviations: DFSP, dermatofibrosarcoma protuberans; FISH, fluorescence in situ hybridization. tumors (94\%) showed a diffuse staining with the CD34 antibody and $2(6 \%)$ a focal staining. These non-interpretable FISH cases were not included in the following sections and percentages.

\section{Certain Diagnosis of Dermatofibrosarcoma Protuberans}

Tumor specimens from 185 patients (93 females and 92 males) were considered as certain according to clinical, histologic, and immunohistochemical examinations. The median age of the patients was 41 years (range 3-89 years). The specimens were located: 77 $(42 \%)$ in the trunk wall (21 in the back, 41 in the chest wall, and 17 in the abdominal wall), $34(18 \%)$ in the upper limb (18 in the shoulder, 10 in the collarbone region, 3 in the arm, 2 in the forearm, and 1 in the wrist), 46 (25\%) in the lower limb (13 in the inguinal region, 11 in the thigh, 9 in the leg, 4 in the pubis, 4 in the buttock, 3 in the ankle, and 2 in the foot), 14 (8\%) in the head and neck, and $2(1 \%)$ in the genital area. For $12(6 \%)$ cases, the site was unknown.

Six $(3 \%)$ specimens were obtained via punch biopsy, 11 (6\%) via shave biopsy, and 168 (91\%) via resections.

One hundred and eighty-three (99\%) tumor specimens were positive for CD34 with a diffuse staining, whereas $1(0.5 \%)$ showed a focal staining. For one $(0.5 \%)$ case, the immunohistochemistry test was not performed.

One hundred and seventy-eight (96\%) FISH analyses were positive, 7 (4\%) were negative.

The dermatofibrosarcoma protuberans in this group were divided into four subgroups: classical dermatofibrosarcoma protuberans (160 cases), pigmented dermatofibrosarcoma protuberans (2 cases), fibrosarcomatous dermatofibrosarcoma protuberans (19 cases), and giant cell fibroblastoma (4 cases). The tumor specimens with a negative FISH were classical dermatofibrosarcoma protuberans.

\section{Probable Diagnosis of Dermatofibrosarcoma Protuberans}

Tumor specimens from 114 patients (60 females and 54 males) were allocated into this group after clinical, histologic, and immunohistochemical evaluations before molecular analysis. The median age of the patients was 46 years (range 1-87 years). The tumors occurred: $45(40 \%)$ in the trunk wall (20 in the back, 12 in the abdominal wall, and 13 in the chest wall); $25(22 \%)$ in the upper limb (13 in the shoulder, 7 in the collarbone region, 4 in the arm, and 1 in the hand); 27 (24\%) in the lower limb (11 in the thigh, 7 in the inguinal region, 5 in the leg, 2 in the foot, 1 in the pubis, and 1 in the ankle); 9 $(8 \%)$ in the head and neck; and $1(1 \%)$ in the genital area. For 7 (5\%) cases, the site was unknown.

Analyzed specimens were obtained via punch biopsies $(n=13 ; 11 \%)$, shave biopsies $(n=17 ; 15 \%)$ (Figure 1), and resection $(n=84 ; 73 \%)$. 
One hundred and thirteen cases (99\%) showed a diffuse staining with the CD34 antibody and 1 (1\%) case exhibited a focal staining.

Of the 114 cases qualified as probable, FISH analysis revealed the specific COL1A1/PDGFB translocation in 104 (91\%) cases (Figure 2), confirming the dermatofibrosarcoma protuberans diagnosis. In $10(9 \%)$ cases, the test did not detect any genetic rearrangement and the final diagnosis was low-grade myofibroblastic sarcoma, dermatofibroma, spindle cell lipoma, and perineurioma in one case each, and undifferentiated sarcoma and solitary fibrous tumor in two cases each. For two cases, the diagnosis of dermatofibrosarcoma protuberans was maintained.

\section{Possible Diagnosis of Dermatofibrosarcoma Protuberans}

Tumor specimens of 112 patients ( 51 females and 61 males) with a median age of 46 years (range 6-86
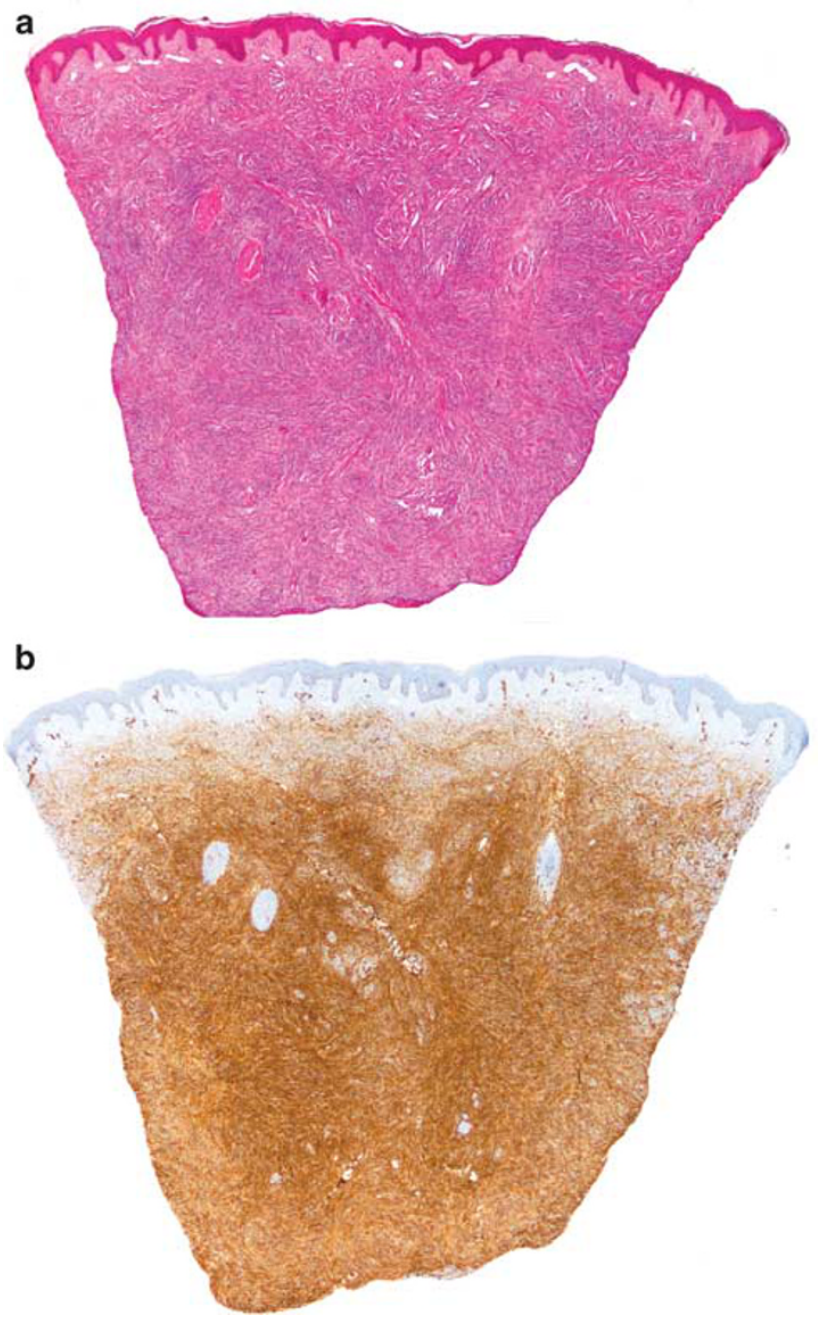

Figure 1 Tumor specimen obtained via shaving biopsy considered as probable dermatofibrosarcoma protuberans. (a) HES; and (b) CD34 (magnification $\times 2.5$ ). years) were diagnosed as possible dermatofibrosarcoma protuberans before molecular analysis. The tumors occurred: $32(26 \%)$ in the trunk wall (17 in the back, 13 in the chest wall, and 2 in the abdominal wall); 35 (31\%) in the upper limb (14 in the shoulder, 9 in the arm, 6 in the hand, 5 in the forearm, and 1 in the collarbone region); 27 (25\%) in the lower limb (6 in the thigh, 7 in the leg, 4 in the foot, 3 in the inguinal region, 3 in the buttock, 2 in the knee, and 2 in the pubis); 15 (13\%) in the head and neck; and $2(2 \%)$ in the genital area. For $3(4 \%)$ cases, the site was unknown.

The biopsy method was punch in $12(10 \%)$ cases, shave in $12(10 \%)$ cases, and resection in $88(80 \%)$ cases.

Eighty-eight cases (80\%) showed a diffuse staining with the CD34 antibody, 16 (13\%) showed a focal staining, and $8(7 \%)$ were negative for this marker.

In the group of 112 cases classified as possible before molecular analyses, FISH results were negative in 91 (81\%) cases, positive in 21 (19\%) cases.

Table 2 shows the initial diagnosis of these 21 molecularly confirmed dermatofibrosarcoma protuberans cases. Initial diagnoses before FISH analysis were: sarcoma in 12 cases (Figure 3), benign lesion in 8 cases (Figure 4), and tumor of intermediate malignancy in 1 case. CD 34 staining was diffusely positive in $18(86 \%)$ cases, focally positive in 2 $(10 \%)$ cases, and negative in $1(4 \%)$ case (initially considered as myxofibrosarcoma). In 11 (52\%) cases, tissue sample was obtained by resection, in $4(20 \%)$ cases by shave biopsy, and in $6(28 \%)$ cases by punch biopsy.

\section{Immunohistochemical Findings}

Three hundred and eighty-two (93\%) cases were positive for CD34 with a diffuse staining, 19 (4.5\%)

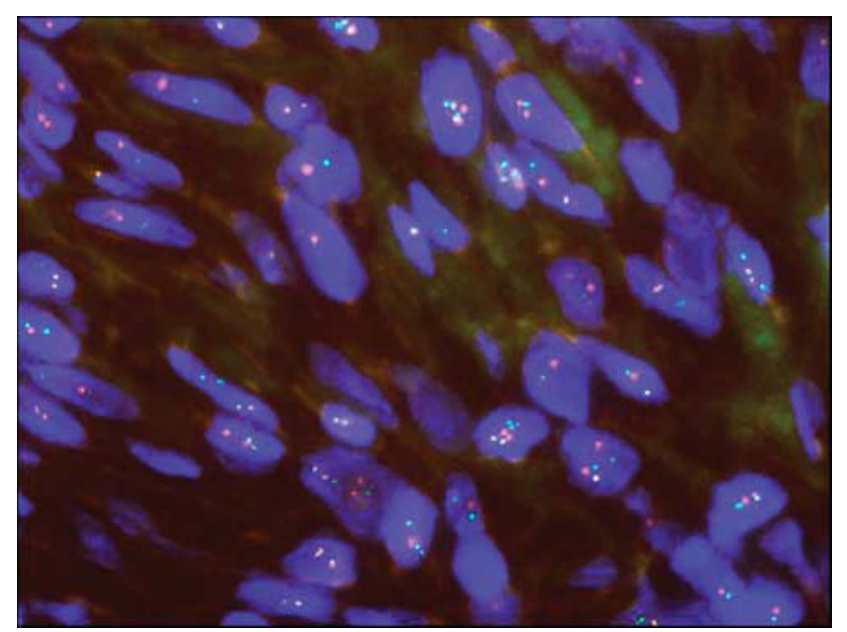

Figure 2 COL1A1/PDGFb rearrangement (fusion signal) detected by fluorescence in situ hybridization in a case considered as probable dermatofibrosarcoma protuberans. 
Table 2 Possible cases with a positive FISH $(n=21)$

\begin{tabular}{ll} 
Initial diagnosis & Final diagnosis \\
\hline Undifferentiated sarcoma $(n=8)$ & $\begin{array}{l}\text { DFSP NOS }(n=4) \\
\text { Fibrosarcomatous DFSP } \\
(n=4)\end{array}$ \\
Myxfibrosarcoma $(n=4)$ & $\begin{array}{l}\text { Myxoid DFSP }(n=4) \\
\text { Dermatofibroma }(n=2)\end{array}$ \\
$\begin{array}{l}\text { DFSP NOS }(n=2) \\
\text { Perineurioma }(n=1)\end{array}$ & DFSP NOS $(n=1)$ \\
malignant evidence $(n=3)$ & DFSP NOS $(n=3)$ \\
Reactive lesion $(n=1)$ & Plaque-like DFSP $(n=1)$ \\
Benign nerve sheath tumor $(n=1)$ & DFSP NOS $(n=1)$ \\
Solitary fibrous tumor $(n=1)$ & DFSP NOS $(n=1)$
\end{tabular}

Abbreviations: DFSP, dermatofibrosarcoma protuberans; FISH, fluorescence in situ hybridization; NOS, not otherwise specified.

cases showed a focal staining, 8 cases $(2 \%)$ were negative, and for $2(0.5 \%)$ cases immunohistochemistry was not performed (Table 3). Cases with a negative CD34 staining pattern were finally diagnosed as undifferentiated sarcoma (five cases), dermatofibrosarcoma protuberans (one case), reactive lesion (one case) and low-grade fibromyxoid sarcoma (one case). Focal staining was observed in dermatofibroma (nine cases), undifferentiated sarcoma (six cases), dermatofibrosarcoma protuberans (three cases), myxofibrosarcoma (one case), and perineurioma (one case). Among the 305 cases finally diagnosed as dermatofibrosarcoma protuberans, $300(99 \%)$ cases were diffusely positive for CD 34,3 showed $(0.8 \%)$ a focal staining, $1(0.2 \%)$ was negative, and for 1 the marker was not done. This staining was observed in others lesions: 19 $(63 \%)$ undifferentiated sarcomas were diffusely CD34 positive, $20(71 \%)$ dermatofibromas showed a diffuse staining and $7(28 \%)$ a peri-lesional staining, and $6(85 \%)$ myxofibrosarcomas and 4 $(100 \%)$ spindle cell lipomas were CD34 positive (diffuse staining).

\section{Size of Sample}

The probable and possible cases were significantly more frequent in the samples obtained through shave and punch biopsies (76\%) than in those obtained via resection (51\%; $P<0.001$; Table 4). Non-interpretable FISH results were equally frequent in resection (9\%) and in shave and punch biopsy $(7 \%)$ specimens.

\section{Discussion}

Most dermatofibrosarcoma protuberans are cytogenetically characterized by the presence of either supernumerary ring chromosomes containing interspersed sequences from chromosomes 17 and 22, or the unbalanced translocation $\mathrm{t}(17 ; 22) .1,5,13,14,19-21$ These rearrangements result in the fusion of the $\alpha 1$ chain of type-1 collagen (COL1A1) gene on chromosome 17 and the platelet-derived growth factor $\beta$ (PDGFB) gene on chromosome 22. The breakpoint in $P D G F B$ gene is constant, placing exon 2 under the control of the COL1A1 promoter. Many COL1A1 gene breakpoints have been identified and proven to be located between exons 7 and 47 (see ref. 14). To date, no correlation between the location of the COL1A1 breakpoint and clinical or pathological features has been established. ${ }^{9}$

These molecular abnormalities are detectable by FISH or by reverse-transcription PCR (RT-PCR). ${ }^{8,10,11,22}$ Given the variability of COL1A1 gene breakpoints, RT-PCR is difficult to perform on paraffin-embedded samples and FISH remains the most appropriate method in routine practice.

In this study, we report a large series of potential dermatofibrosarcoma protuberans for which molecular testing was systematically performed. The aim was to evaluate the diagnostic value of FISH analysis to solve cases with dubious diagnosis. Each tumor specimen was classified into one of the three groups according to the probability of dermatofibrosarcoma protuberans diagnosis. This classification was the main limitation of this study because it is conditioned by the analysis and the experience of the pathologist. To minimize the impact of this issue, the classification was supported by the work of two independent pathologists (one senior and one junior).

This series shows that FISH is not necessary for the diagnosis when assessed as certain by the pathologist: no certain diagnosis was modified following the FISH results. Remarkably, $96 \%$ of the cases with successful FISH were found to harbor the COL1A1/PDGFB translocation. This result is in accordance with previous research, if we consider the series consisted of cases with an histological review, allowing a certain diagnosis, this translocation is described in $96 \%$ of 27 cases, ${ }^{10}$ in $95 \%$ of 47 cases, ${ }^{9}$ and in $85 \%$ of 34 cases (but 6 FISH analysis were non interpretable). ${ }^{8}$ In an other study with histological review, this chromosomal rearrangement is more frequent in fibrosarcomatous dermatofibrosarcoma protuberans $(100 \%)$ than in classical dermatofibrosarcoma protuberans $(76 \%) .12$ This rate is lower in a series of 57 cases, but this series was based on cases without histological review. ${ }^{11}$ Other chromosomal rearrangements have been described in rare cases of dermatofibrosarcoma protuberans, including translocations $\mathrm{t}(5 ; 8), \mathrm{t}(9 ; 22), \mathrm{t}(2 ; 17)$, and $\mathrm{t}(\mathrm{X} ; 7) .{ }^{18,23-25}$ FISH analysis allowed to identify those cases with a certain diagnosis lacking the COL1A1/PDGFB-specific translocation. The identification and the collection of those cases is the first step in the research of potential unknown cytogenetic abnormalities implicated in the pathogenesis of dermatofibrosarcoma protuberans, and could be of great value in the field of diagnosis.

Among the probable cases, FISH confirmed the diagnosis in $91 \%$ of the cases, and led to rectification 

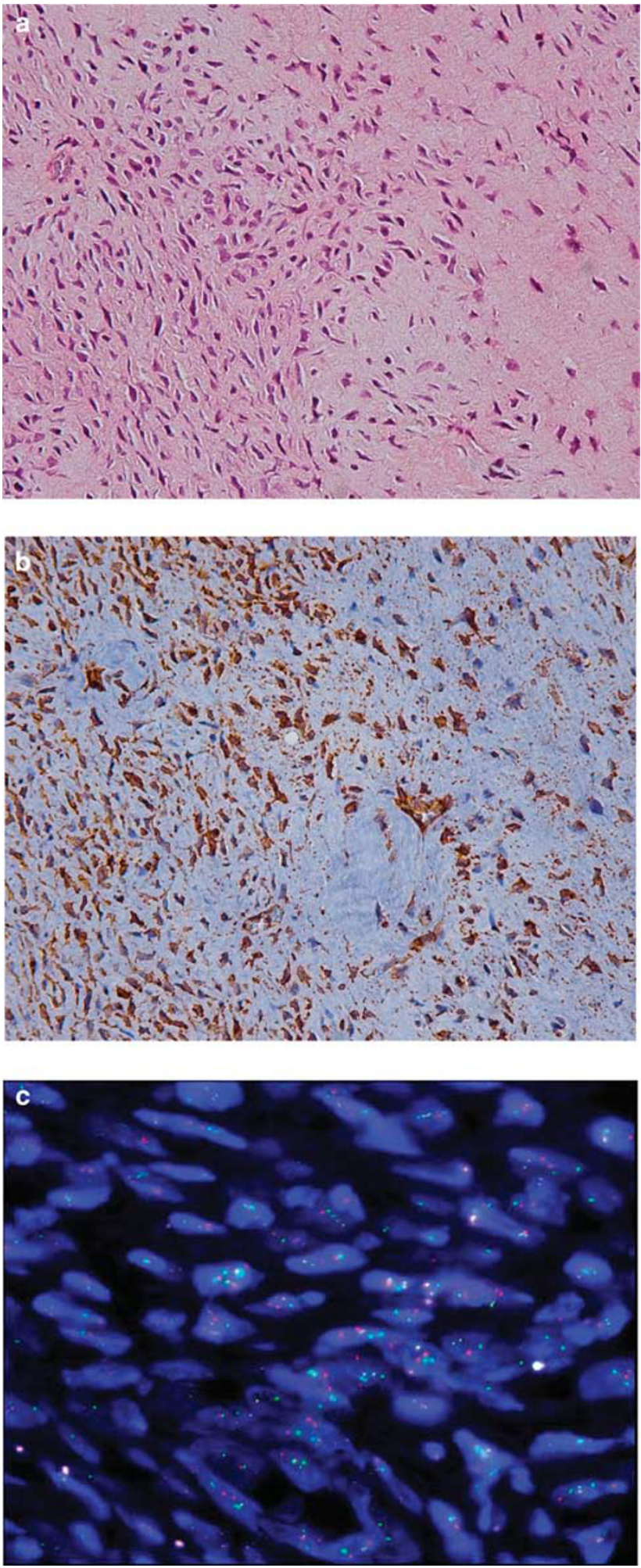

Figure 3 Dermatofibrosarcoma protuberans initially considered as undifferentiated sarcoma. (a) HES; (b) CD34 (magnification $\times 20$ ); and (c) COL1A1/PDGFb rearrangement (fusion signal) detected by fluorescence in situ hybridization.

in $7 \%$ of the cases. When the diagnosis of dermatofibrosarcoma protuberans was classified as possible, FISH was necessary to correlate the diagnosis in
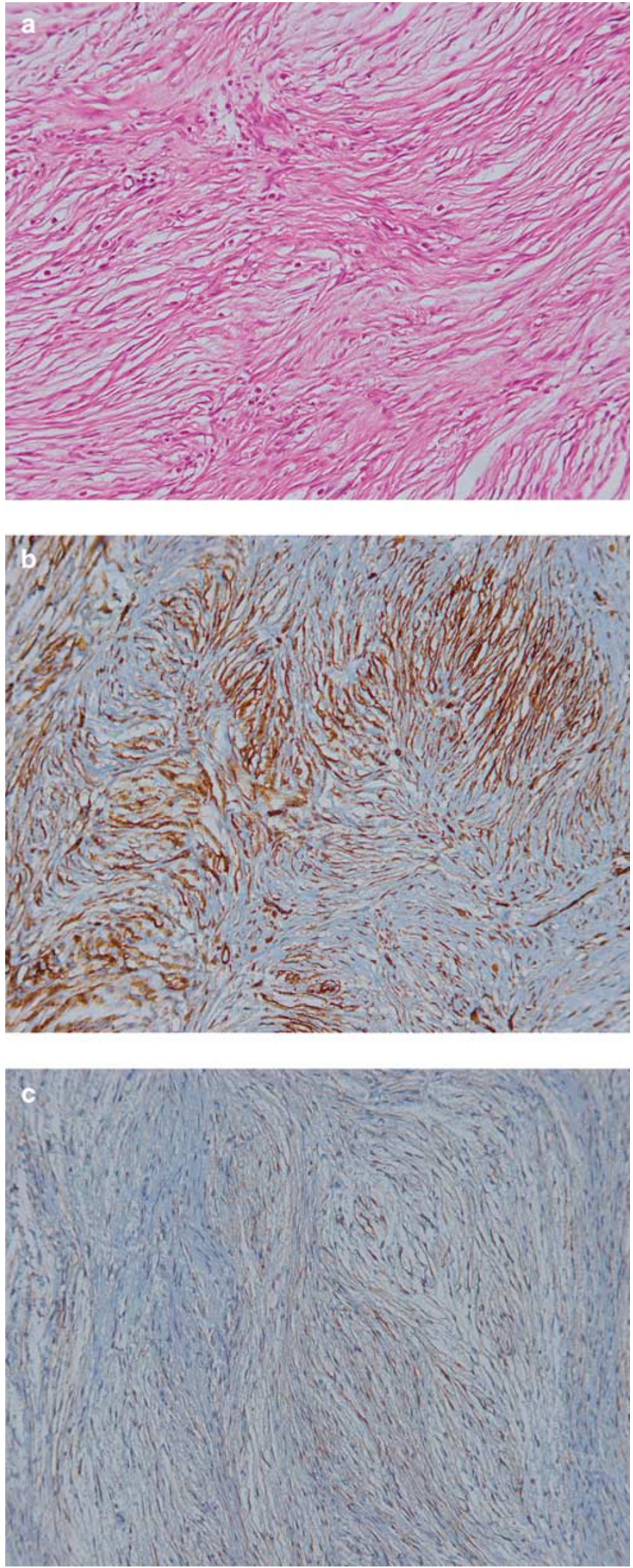

Figure 4 Dermatofibrosarcoma protuberans initially considered as perineurioma. (a) HES; (b) diffuse expression of CD34; and (c) positive immunostaining for epithelial membrane antigen (magnification $\times 20$ ).

$19 \%$ of cases. Therefore, FISH analysis was useful for confirming 104 cases ( $25 \%$ of the whole series) of probable diagnosis and necessary for establishing 
Table 3 CD34 result according to FISH positivity

\begin{tabular}{lrrr}
\hline & FISH + & FISH - & Total \\
\hline CD34 + (diffuse staining) & 298 & 84 & 382 \\
CD34 + (focal staining) & 3 & 16 & 19 \\
CD34 - & 1 & 7 & 8 \\
Not done & 1 & 1 & 2 \\
Total & 303 & 108 & 411 \\
\hline
\end{tabular}

Abbreviation: FISH, fluorescence in situ hybridization.

Table 4 Proportion of the possible/probable cases and certain cases in the shaving/punch biopsies and in the resections

\begin{tabular}{lcrc}
\hline & $\begin{array}{c}\text { Shaving biopsies/ } \\
\text { punch biopsies }\end{array}$ & Resections & $\begin{array}{c}\text { P-value } \\
\left(\chi^{2}\right)\end{array}$ \\
\hline $\begin{array}{l}\text { Possible/probable cases } \\
\text { Certain cases }\end{array}$ & $54(76 \%)$ & $172(51 \%)$ & $<0.001$ \\
Total & $17(24 \%)$ & $168(49 \%)$ & \\
& 71 & 340 & \\
\hline
\end{tabular}

the diagnosis of dermatofibrosarcoma protuberans of 21 cases (5\% of the whole series) of possible diagnosis. In a previous study, ${ }^{17}$ the role of FISH analysis in the diagnosis of dermatofibrosarcoma protuberans was limited because the group of dermatofibrosarcoma protuberans cases was smaller than that in this series.

The differential diagnoses of dermatofibrosarcoma protuberans usually mentioned include dermatofibroma, nodular fasciitis, fibroxanthoma, neurofibroma, and myxoid liposarcoma. ${ }^{4,26}$ In this large series of potential dermatofibrosarcoma protuberans, other differential diagnoses emerged. The analysis of probable cases has shown that perineurioma, benign nerve sheath tumor, solitary fibrous tumor and undifferentiated sarcoma could mimic dermatofibrosarcoma protuberans. The possible cases with a positive FISH have shown that dermatofibrosarcoma protuberans could display very different appearance and looked like reactive lesion, perineurioma, undifferentiated tumor without malignant evidence, benign nerve sheath tumor, solitary fibrous tumor, undifferentiated sarcoma and myxofibrosarcoma.

Immunohistochemical staining can be very convenient to distinguish dermatofibrosarcoma protuberans from other tumors and to confirm diagnosis. CD34 was found to have an excellent sensitivity of $99 \%$. In others studies, the sensitivity of this test varied from 84 to $100 \% .{ }^{26-28}$ The test was less successful in differentiating dermatofibrosarcoma protuberans from its main differential diagnoses, including $19(63 \%)$ undifferentiated sarcomas, 6 $(85 \%)$ myxofibrosarcomas, and $4(100 \%)$ spindle cell lipomas stained positive for CD34. Kim et al. ${ }^{28}$ found CD34 to be a valuable stain for differentiating between dermatofibrosarcoma protuberans and dermatofibroma, and reported a specificity of $83 \%$.
In our study, the specificity of CD34 was particularly low with $28(71 \%)$ dermatofibromas showing a diffuse staining and $7(28 \%)$ a focal staining. This result is explained by the inclusion in our study of a high number of specific dermatofibromas that mimic - and are therefore difficult to differentiate from-dermatofibrosarcoma protuberans. Thus, with an excellent sensibility, CD34 is an essential test to support the histological diagnosis, but in series like ours insufficient because of its low specificity.

FISH analysis can be necessary, not only to obtain an accurate diagnosis, but also to determine the most appropriate treatment. Dermatofibrosarcoma protuberans is a locally aggressive intermediate malignancy tumor, rarely metastasizing but with a risk of local recurrences, requiring a surgical excision with wide resection margins. The distinction between dermatofibrosarcoma protuberans and a benign lesion that mimics this tumor is essential. Therefore, for advanced cases or cases with difficult excision, a targeted therapy with tyrosine kinase inhibitor (imatinib mesylate) has been approved. ${ }^{1-3,29}$ The COL1A1-PDGFB chimeric protein seems to be involved in the activation of the PDGF receptor. ${ }^{2}$ In this context, the detection of the chromosomal rearrangement involving $P D G F B$ is essential to favor this treatment. ${ }^{30}$

In this study, the cases with probable or possible diagnoses were significantly more frequent in punch and shaving biopsies than in resection samples. As small biopsies are currently recommended for diagnosis in soft tissue tumor, ${ }^{31}$ the value of the molecular tests increases in this scenario.

In summary, FISH analysis was useful in confirming a diagnosis of dermatofibrosarcoma protuberans in $25 \%$ of the cases, and necessary for $5 \%$ of the cases. Nevertheless, in most cases, histology with immunohistochemistry remains the gold standard for establishing an accurate diagnosis.

\section{Acknowledgments}

Ninety-five cases were part of the GENSARC 2008 study: 'Medical Economic Evaluation of the Molecular Detection by FISH (Fluorescent in Situ Hybridization) and by PCR (Polymerase Chain Reaction) of Sarcomas Specific Translocations and Amplifications' (NCT0084769) supported by the French National Cancer Institute (INCa). This work was supported by SIRIC BRIO (Site de Recherche Intégrée sur le Cancer - Bordeaux Recherche Intégrée Oncologie) (Grant INCa-DGOS-Inserm 6046). We warmly thank Jone Iriondo of Institut Bergonié for providing medical editorial assistance.

\section{Disclosure/conflict of interest}

The authors declare no conflict of interest. 


\section{References}

1 Mentzel T, Pedeutour F, Lazar AJF, et al. Dermatofibrosarcoma protuberans. In: Fletcher CDM, Bridge JA, Hogendoorn PCW (eds). World Health Organisation Classification of Tumours of Soft Tissue and Bone. IARC Press: Lyon, France; 2013, pp 77-79.

2 Liu LS, Colegio OR. Molecularly targeted therapies for nonmelanoma skin cancers. Int J Dermatol 2013;52: 654-665.

3 Rubin BP, Schuetze SM, Eary JF, et al. Molecular targeting of platelet-derived growth factor B by imatinib mesylate in a patient with metastatic dermatofibrosarcoma protuberans. J Clin Oncol 2002;20:3586-3591.

4 Weiss S, Goldblum JR. Dermatofibrosarcoma protuberans, In: Weiss SW, Goldblum JR (eds). Enzinger and Weiss's Soft Tissue Tumors. Mosby: St Louis, MO, USA; 2008, pp 371-382.

5 Pedeutour F, Simon MP, Minoletti F, et al. Ring 22 chromosomes in dermatofibrosarcoma protuberans are low-level amplifiers of chromosome 17 and 22 sequences. Cancer Res 1995;55:2400-2403.

6 Pedeutour F, Simon MP, Minoletti F, et al. Translocation, $\mathrm{t}(17 ; 22)(\mathrm{q} 22 ; \mathrm{q} 13)$, in dermatofibrosarcoma protuberans: a new tumor-associated chromosome rearrangement. Cytogenet Cell Genet 1996;72:171-174.

7 Simon MP, Pedeutour F, Sirvent N, et al. Deregulation of the platelet-derived growth factor B-chain gene via fusion with collagen gene COL1A1 in dermatofibrosarcoma protuberans and giant-cell fibroblastoma. Nat Genet 1997;15:95-98.

8 Segura S, Salgado R, Toll A, et al. Identification of $\mathrm{t}(17 ; 22)(\mathrm{q} 22 ; \mathrm{q} 13)$ (COL1A1/PDGFB) in dermatofibrosarcoma protuberans by fluorescence in situ hybridization in paraffin-embedded tissue microarrays. Hum Pathol 2011;42:176-184

9 Ha SY, Lee SE, Kwon MJ, et al. PDGFB rearrangement in dermatofibrosarcoma protuberans: correlation with clinicopathologic characteristics and clinical implications. Hum Pathol 2013;44:1300-1309.

10 Patel KU, Szabo SS, Hernandez VS, et al. Dermatofibrosarcoma protuberans COL1A1-PDGFB fusion is identified in virtually all dermatofibrosarcoma protuberans cases when investigated by newly developed multiplex reverse transcription polymerase chain reaction and fluorescence in situ hybridization assays. Hum Pathol 2008;39:184-193.

11 Takahira T, Oda Y, Tamiya S, et al. Detection of COL1A1-PDGFB fusion transcripts and PDGFB/ PDGFRB mRNA expression in dermatofibrosarcoma protuberans. Mod Pathol 2007;20:668-675.

12 Llombart B, Monteagudo C, Sanmartín O, et al. Dermatofibrosarcoma protuberans: a clinicopathological, immunohistochemical, genetic (COL1A1-PDGFB), and therapeutic study of low-grade versus high-grade (fibrosarcomatous) tumors. J Am Acad Dermatol 2011;65:564-575.

13 Minoletti F, Miozzo M, Pedeutour F, et al. Involvement of chromosomes 17 and 22 in dermatofibrosarcoma protuberans. Genes Chromosomes Cancer 1995;13:62-65.

14 Sirvent N, Maire G, Pedeutour F. Genetics of dermatofibrosarcoma protuberans family of tumors: from ring chromosomes to tyrosine kinase inhibitor treatment. Genes Chromosomes Cancer 2003;37:1-19.

15 Antonescu CR. The role of genetic testing in soft tissue sarcoma. Histopathology 2006;48:13-21.
16 Cheah AL, Billings SD. The role of molecular testing in the diagnosis of cutaneous soft tissue tumors. Semin Cutan Med Surg 2012;31:221-233.

17 Neuville A, Ranchère-Vince $\mathrm{D}$, Dei Tos AP, et al. Impact of molecular analysis on the final sarcoma diagnosis: a study on 763 cases collected during a European epidemiological study. Am J Surg Pathol 2013;37:1259-1268.

18 Bianchini L, Maire G, Guillot B, et al. Complex t(5;8) involving the CSPG2 and PTK2B genes in a case of dermatofibrosarcoma protuberans without the COL1A1-PDGFB fusion. Virchows Arch 2008;452: 689-696.

19 Mandahl N, Limon J, Mertens F, et al. Ring marker containing $17 \mathrm{q}$ and chromosome 22 in a case of dermatofibrosarcoma protuberans. Cancer Genet Cytogenet. 1996;89:88-91.

20 Naeem R, Lux ML, Huang SF, et al. Ring chromosomes in dermatofibrosarcoma protuberans are composed of interspersed sequences from chromosomes 17 and 22 . Am J Pathol 1995;147:1553-1558.

21 Dobin SM, Diaz JA, Silva MT, et al. Translocation $(17 ; 22)(q 22 ; q 13)$ in a case of subcutaneous dermatofibrosarcoma protuberans in an adult. Cancer Genet Cytogenet 1999;109:86-87.

22 Kutzner H, Mentzel T, Palmedo G, et al. Plaque-like CD34-positive dermal fibroma ("medallion-like dermal dendrocyte hamartoma"): clinicopathologic, immunohistochemical, and molecular analysis of 5 cases emphasizing its distinction from superficial, plaquelike dermatofibrosarcoma protuberans. Am J Surg Pathol 2010;34:190-201.

23 Sonobe H, Furihata M, Iwata J, et al. Dermatofibrosarcoma protuberans harboring $\mathrm{t}(9 ; 22)(\mathrm{q} 32 ; \mathrm{q} 12.2)$. Cancer Genet Cytogenet 1999;110:14-18.

24 Sinovic J, Bridge JA. Translocation $(2 ; 17)$ in recurrent dermatofibrosarcoma protuberans. Cancer Genet Cytogenet 1994;75:156-157.

25 Craver RD, Correa H, Kao Y, et al. Dermatofibrosarcoma protuberans with $46, \mathrm{XY}, \mathrm{t}(\mathrm{X} ; 7)$ abnormality in a child. Cancer Genet Cytogenet 1995;80:75-77.

26 Bogucki B, Neuhaus I, Hurst EA. Dermatofibrosarcoma protuberans: a review of the literature. Dermatol Surg 2012;38:537-551.

27 Haycox CL, Odland PB, Olbricht SM, et al. Immunohistochemical characterization of dermatofibrosarcoma protuberans with practical applications for diagnosis and treatment. J Am Acad Dermatol 1997;37: 438-444.

28 Kim HJ, Lee JY, Kim SH, et al. Stromelysin-3 expression in the differential diagnosis of dermatofibroma and dermatofibrosarcoma protuberans: comparison with factor XIIIa and CD34. Br J Dermatol 2007;157: 319-324.

29 Kérob D, Porcher R, Vérola O, et al. Imatinib mesylate as a preoperative therapy in dermatofibrosarcoma: results of a multicenter phase II study on 25 patients. Clin Cancer Res 2010;16:3288-3295.

30 Kerob D, Pedeutour F, Leboeuf C, et al. Value of cytogenetic analysis in the treatment of dermatofibrosarcoma protuberans. J Clin Oncol 2008;26: 1757-1759.

31 Ray-Coquard I, Ranchère-Vince D, Thiesse $\mathrm{P}$, et al. Evaluation of core needle biopsy as a substitute to open biopsy in the diagnosis of soft-tissue masses. Eur J Cancer 2003;39:2021-2025. 